\title{
Evaluation of the linear interpolation method in correcting the influence of slice thicknesses on radiomic feature values in solid pulmonary nodules: a prospective patient study
}

\author{
Shouxin Yang ${ }^{1}$, Ning $\mathrm{Wu}^{1,2}$, Li Zhang ${ }^{1}$, Meng $\mathrm{Li}^{1}$ \\ ${ }^{1}$ Department of Diagnostic Radiology, National Cancer Center/National Clinical Research Center for Cancer/Cancer Hospital, Chinese Academy of \\ Medical Sciences and Peking Union Medical College, Beijing, China; ${ }^{2}$ PET-CT Center, National Cancer Center/National Clinical Research Center \\ for Cancer/Cancer Hospital, Chinese Academy of Medical Sciences and Peking Union Medical College, Beijing, China \\ Contributions: (I) Conception and design: N Wu, L Zhang, S Yang; (II) Administrative support: N Wu; (III) Provision of study materials or patients: \\ S Yang; (IV) Collection and assembly of data: S Yang, M Li, L Zhang; (V) Data analysis and interpretation: S Yang, L Zhang, M Li; (VI) Manuscript \\ writing: All authors; (VII) Final approval of manuscript: All authors. \\ Correspondence to: Ning Wu. Department of Diagnostic Radiology/ PET-CT Center, National Cancer Center/National Clinical Research \\ Center for Cancer/Cancer Hospital, Chinese Academy of Medical Sciences and Peking Union Medical College, Beijing 100021, China. \\ Email: cjr.wuning@vip.163.com; Li Zhang. Department of Diagnostic Radiology, National Cancer Center/National Clinical Research Center for \\ Cancer/Cancer Hospital, Chinese Academy of Medical Sciences and Peking Union Medical College, Beijing 100021, China. Email: tjmuzl77@163.com.
}

Background: To investigate the influence of slice thickness on radiomic feature (RF) values in solid pulmonary nodules and evaluate the effect of a linear interpolation method in correcting the influence.

Methods: Thirty pulmonary nodules from 28 patients were selected prospectively with a thick-slice of $5 \mathrm{~mm}$ and a thin-slice of $1.25 \mathrm{~mm}$ on CT. A resampling method was used to normalize the voxel size of thick and thin slices CT images to $1 \times 1 \times 1 \mathrm{~mm}^{3}$ by linear interpolation. Lung nodules were segmented manually. A total of 396 radiomic features (RFs) were extracted from thick-slice and thin-slice images, together with the images resampled from thick (thick-r) and thin (thin-r) slices. The differences between the RF values were evaluated using a paired $t$-test. A comparison between groups was made using the Chi-squared test.

Results: Among the 396 RFs, 305 RFs showed an intraclass correlation coefficient $\geq 0.75$ after test-retest analysis (including 22 histogram features, 20 geometry features, and 263 texture features). In the nonresampled data, $239 \mathrm{RF}$ values $(78.4 \%$, 239/305) showed significant differences between thick and thin slice CT images. Resampling of thick images revealed that 202 RF values (66.2\%, 202/305) showed significant differences between thick-r and thin slice CT images, showing a significant decrease in the number of different $\mathrm{RF}$ values when compared to non-resampled data $(\mathrm{P}<0.01)$. For the RF subgroups, only texture features showed a significant reduction in the number of different $\mathrm{RF}$ values after resampling $(\mathrm{P}<0.01)$. When both thick and thin slice images were resampled, the number of significantly different RF values between thick-r and thin-r images was increased to 247 (81.0\%, 247/305), showing no significant difference when compared to non-resampled data $(\mathrm{P}=0.421)$.

Conclusions: Slice thickness demonstrated a considerable influence on RF values in solid pulmonary nodules, producing false results when CT images with different slice thicknesses were used. Linear interpolation of the resampling method was limited because of the relatively small correction effect.

Keywords: Linear interpolation; radiomics; slice thickness; pulmonary nodules; voxel size

Submitted Mar 31, 2020. Accepted for publication Nov 19, 2020.

doi: 10.21037/atm-20-2992

View this article at: http://dx.doi.org/10.21037/atm-20-2992 


\section{Introduction}

Radiomics, an evolving research field, was first proposed by Dutch scholars in 2012 (1). It refers to a high-throughput quantitative analysis that extracts hundreds of quantitative imaging features from the area of interest, followed by screening and analysis to describe information such as biological characteristics and tumor heterogeneity (1). The radiomic research process mainly includes image acquisition and reconstruction, image segmentation, feature extraction, and analysis (2). As part of the development of new imaging approaches, radiomics has been introduced as a post-processing computational approach to qualitatively and quantitatively associate the imaging data with clinical endpoints (3-6). To date, radiomics has made promising progression in detecting pulmonary nodules (7), diagnosing small solid pulmonary nodules (8), classifying small cell lung cancer and non-small-cell lung cancer (9), and predicting the response of lung cancer to radiotherapy (10).

However, establishing the final image model is influenced by many factors, including computed tomography (CT) scanners, tube voltage, tube current, radiation dose, layer thickness, and other scanning parameters. Few previous studies have confirmed that the parameters associated with CT scans affect the reproducibility of radiomic features (RFs) (11-13). Slice thickness is one of the factors that affect the reproducibility of RFs (14). In addition, $\mathrm{He}$ et al. (15) demonstrated that RFs obtained from thin-slice $(1.25 \mathrm{~mm})$ CT images showed better diagnostic ability than thick-slice $(5 \mathrm{~mm}) \mathrm{CT}$ images. These results implied that thin-slice images play a more meaningful role in radiomic studies. However, the acquisition and management of thinslice CT images require more substantial storage space. Also, the research on image resampling is mainly obtained from retrospective studies. The scanning conditions and other parameters of these studies are difficult to control accurately, generating unknown and uncontrollable factors.

Several studies have reported that the resampling method could improve the reproducibility of RFs (16-19). In a phantom-based study, Shafiq-Ul-Hassan et al. (17) resampled the phantom images to a specific voxel size of $1 \times 1 \times 2 \mathrm{~mm}^{3}$ using linear interpolation. They concluded that resampling was an appropriate preprocessing method to decrease the number of different radiomic feature (RF) values and obtain more reproducible RFs. Resampling by linear interpolation is one of the simplest and the most common preprocessing methods in radiomic studies. Previous studies on resampling were mostly phantom studies and could not reflect the biological information contained in the lesions. Other studies that used patient images for resampling were mainly retrospective. The scanning conditions and other parameters were difficult to control accurately, generating unknown and uncontrollable factors.

To cover this gap, patients' pulmonary nodules were selected prospectively to ensure that the biological information in the nodules could be obtained and to reduce the influence of other scanning factors on the results. Thus, a prospective patient study was conducted to investigate the influence of slice thicknesses on RF values in solid pulmonary nodules and evaluate the effect of a linear interpolation method in correcting the influence.

We present the following study in accordance with the STROBE reporting checklist (available at http://dx.doi. org/10.21037/atm-20-2992).

\section{Methods}

\section{Patients}

The study was conducted in accordance with the Declaration of Helsinki (as revised in 2013), and was approved by the medical ethics committee of National Cancer Center/Cancer Hospital, Chinese Academy of Medical Sciences and Peking Union Medical College (NO.: 19-018/1840). Informed consent was obtained from all patients. This study was designed as a prospective study. A total of 30 solid pulmonary nodules from 28 patients who underwent CT examination in our hospital from 2018 July to December 2018 were included in this study (Figure 1). The inclusion criteria were as follows: (I) patients who were clinically diagnosed with pulmonary nodules and accepted enhanced CT examination in our hospital; (II) CT images of patients showing at least one well-defined solid pulmonary nodule; and (III) the average diameter of the nodule was between 5 and $30 \mathrm{~mm}$. The exclusion criteria were as follows: (I) no solid lung nodule of sufficient size in the lungs; and (II) difficulty in accurately defining the nodule boundaries.

\section{CT image acquisition}

All collected CT images were obtained from a 256-MDCT (Revolution CT, GE Healthcare, Milwaukee, WI, USA). The CT scan parameters were as follows: A tube voltage of $120 \mathrm{kVp}$, a tube current of 50-500 mA, and a pitch of 0.992 . CT images were reconstructed using a standard algorithm, and the slice thicknesses included a thick-slice of $5 \mathrm{~mm}$ and 
a thin-slice of $1.25 \mathrm{~mm}$. CT scans ranged from the thoracic entrance to the bottom of the lung. A contrast medium of $85 \mathrm{ml}$ was intravenously injected at a flow rate of $3 \mathrm{~mL} / \mathrm{s}$, and scanning was performed after a delay of 35 seconds.

\section{Resampling methods}

The simple linear interpolation method was used to resample the thickness of both thick-slice $(5 \mathrm{~mm})$ and thin-

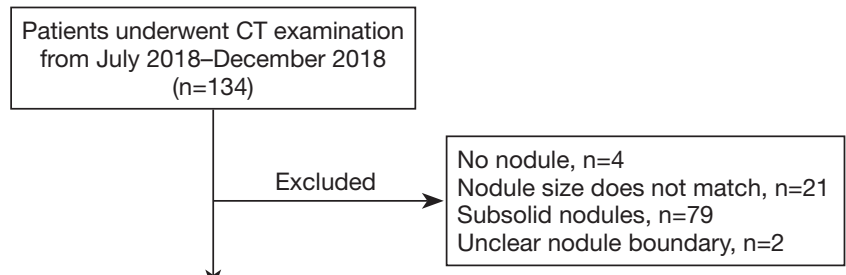

Final entry patients $\mathrm{n}=28$ (30 nodules)

Figure 1 Flow diagram of patient selection. slice $(1.25 \mathrm{~mm})$ images of 30 solid pulmonary nodules. The thick-r images (images after thick-slice images were resampled) and thin-r images (images after thin-slice images were resampled) were then obtained.

\section{Nodule segmentation and feature extraction}

All nodules were segmented manually by a radiologist (S.X.Y) with 5 years' experience using the software ITKSNAP (http://www.itksnap.org/) (20). Segmentation included the whole nodules that started from the first layer and continued to the upper tier where the nodules disappeared (Figure 2, Figure 3). In addition, the segmentation of each layer of the image should include the largest edge of the nodules. Besides, the thin-slice CT images of the 30 solid pulmonary nodules were segmented again by the same radiologist for a consistency test with an interval of no less than 2 weeks.

The extraction and analysis of RFs were carried out using Artificial Intelligence Kit (A.K.) software provided by the GE Healthcare Life Sciences Company (Chicago,
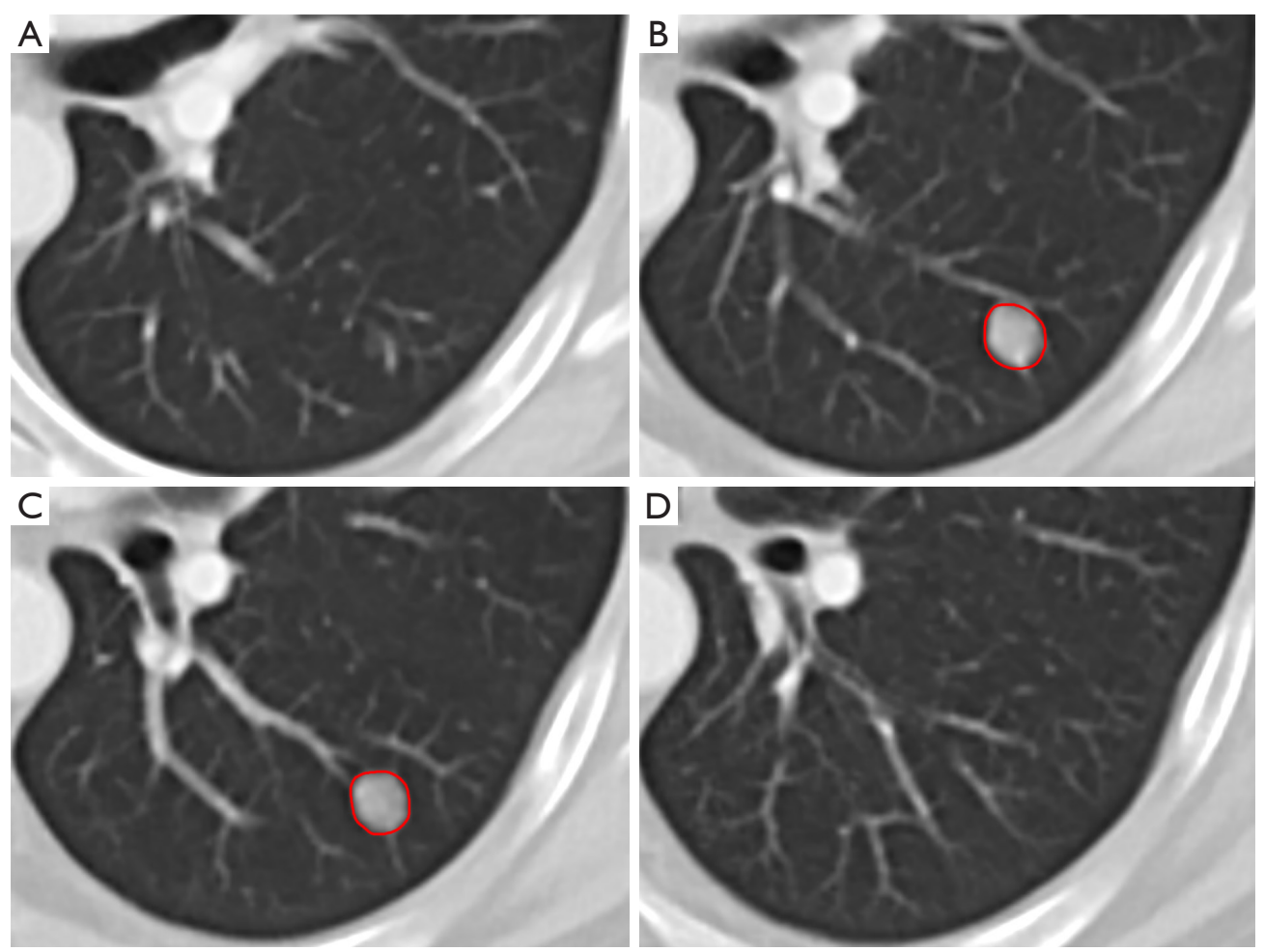

Figure 2 A 57-year-old male with a solid nodule on the left lower lobe. The long-diameter of the nodule is $12.1 \mathrm{~mm}$, and the short-diameter of the nodule is $10.4 \mathrm{~mm}$. Thick slice $(5 \mathrm{~mm}$ ) CT images of the solid nodule were segmented using the ITK-SNAP software (A-D). The red circles represent the ROI boundaries of the nodule. 

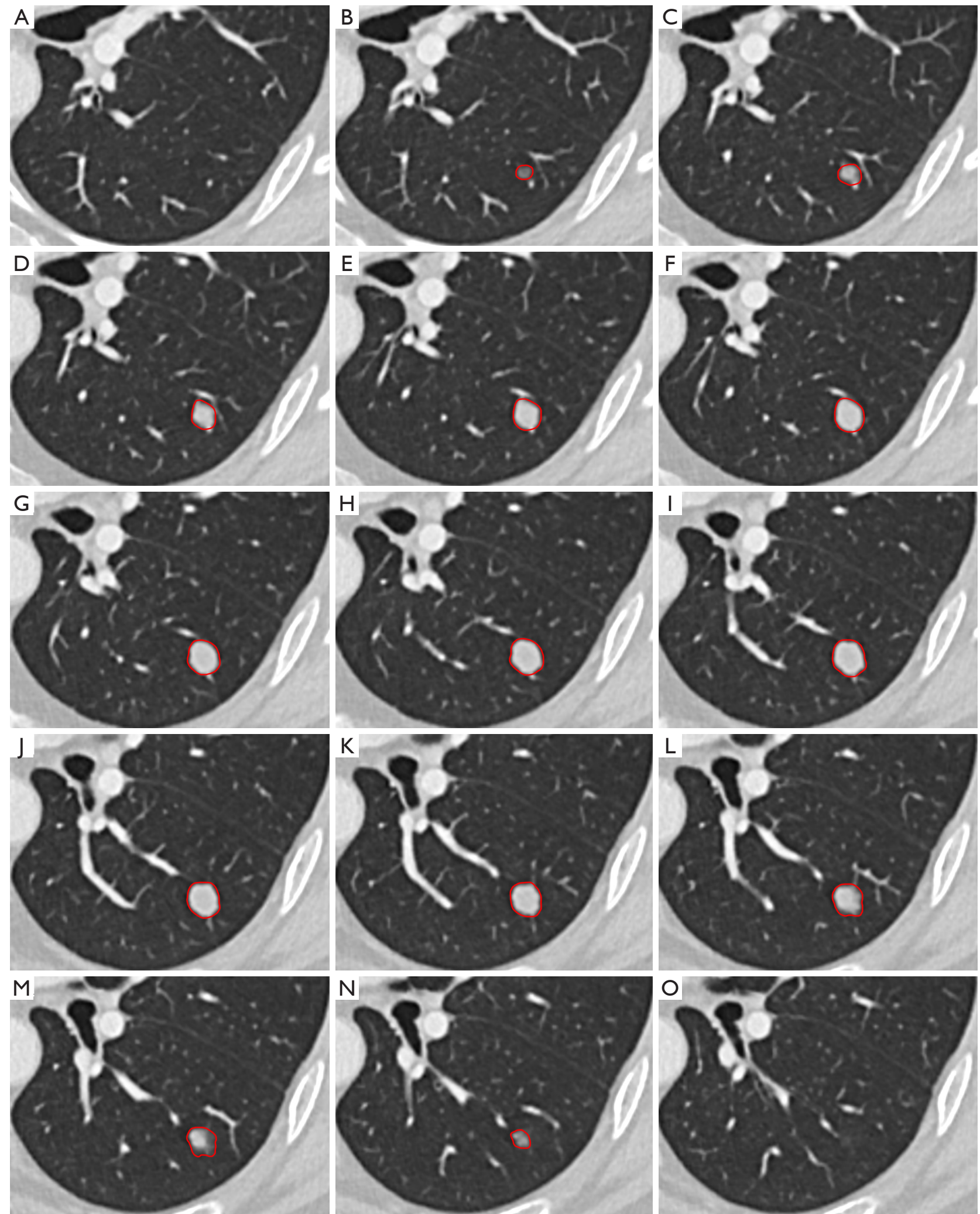

Figure 3 The same nodule from Figure 2. Thin slice $(1.25 \mathrm{~mm})$ CT images of the solid nodule were segmented using the ITK-SNAP software (A-O). The red circles represent the ROI boundaries of the nodule. 
Table 1 Characteristics of 28 patients and 30 pulmonary nodules

\begin{tabular}{lc}
\hline Factors & Outcome \\
\hline Age, mean $\pm \mathrm{SD}$, years & $54.6 \pm 10.7$ \\
Sex, $\mathrm{n}(\%)$ & \\
Male & $12(42.9)$ \\
Female & $16(57.1)$ \\
Size, mean $\pm \mathrm{SD}, \mathrm{mm}$ & $9.3 \pm 3.5$ \\
Location, $\mathrm{n}(\%)$ & \\
RUL & $7(23.3)$ \\
RML & $4(13.3)$ \\
RLL & $4(13.3)$ \\
LUL & $9(30.0)$ \\
LLL & $6(20.0)$ \\
\hline
\end{tabular}

RUL, right upper lobe; RML, right middle lobe; RLL, right lower lobe; LUL, left upper lobe; LLL, left lower lobe.

IL, USA). Initially, all CT images (including the original CT images and the resampled CT images) and the region of interest (ROI) images of nodules were loaded in batches into the A.K. software. This was followed by choosing the features, including the histogram features, geometry features, and texture features. Thereafter, 396 RFs in total were extracted from the software.

\section{Statistical analysis}

The sample size was calculated by the "tests for paired means (simulation)" in PASS 2011 (NCSS, LLC, East Kaysville, UT, USA), and the parameters were set as $\alpha$ $=0.05, \beta=0.10$; distribution parameters $\mathrm{M} 0=0, \mathrm{M} 1=1$; correlation of items $=0.2$; and simulations $=2,000$. The results showed that if the sample size was set to 20 , then the power would be 0.911 ; and if the sample size was set to 30 , then the power would be 0.988 . Therefore, the sample size of our current study met the criteria for statistical power for analysis.

All statistical analyses in this study were performed using SPSS (version 23, IBM Corp., Armonk, NY, USA) software. The intraclass correlation coefficient was used to evaluate the repeatability of the RFs. An intraclass correlation coefficient (ICC) of $\geq 0.75$ was considered to show good repeatability. The differences between the RF values were assessed using a paired $t$ test. A chi-squared test was used for comparisons between the groups. $\mathrm{P}<0.05$ was considered statistically significant.

\section{Results}

\section{Clinical characteristics and feature selection}

A total of 134 patients were scanned initially. Next, 4 cases without nodules, 21 cases with non-matching nodule size, 79 cases with subsolid nodules, and 2 cases with unclear nodule boundaries were excluded. Finally, 28 patients with a total of 30 nodules were enrolled in the study. The characteristics of the patients and their nodules are presented in Table 1. Among the $396 \mathrm{RFs}$, there were $305 \mathrm{RFs}$ with an ICC $\geq 0.75$ after test-retest analysis. The results were calculated using these $305 \mathrm{RFs}$.

\section{Differences in the number of $R F$ values between different comparison groups}

In the non-resampled data, $239 \mathrm{RF}$ values (78.4\%, 239/305) showed significant differences between thickslice and thin-slice CT images of solid pulmonary nodules and $66 \mathrm{RF}$ values $(21.6 \%, 66 / 305)$ remained consistent. When the thick images were resampled, $202 \mathrm{RF}$ values $(66.2 \%, 202 / 305)$ showed significant differences between the thick-r and thin slice CT images of solid pulmonary nodules and 103 (33.8\%, 103/305) remained consistent. The decrease in the number of different RF values between thick $v s$. thin and thick-r $v s$. thin showed significant differences $(\mathrm{P}<0.01$; Table 2$)$. When both the thick and thin slice images were resampled, the significantly different RF values between thick-r and thin-r images increased to $247(81.0 \%, 247 / 305)$ and 58 (19.0\%, $58 / 305) \mathrm{RF}$ values remained consistent. There was a slight increase (from 239 to 247) in different RF values between thick $v s$. thin and thick-r $v s$. thin-r; however, the difference was not statistically significant $(\mathrm{P}=0.421$; Table 2).

When significantly different RFs in the non-resampled data and resampled data were analyzed by subgroups, the texture features showed the least proportion of significantly different RFs, whether in the non-resampled data or the resampled data $(75.7 \%, 61.2 \%$, and $78.7 \%$ in nonresampled data, thick-slice images resampled data, and both images resampled data respectively), while $95.0 \%, 100.0 \%$ and, $95.0 \%$ for geometry features and $95.5 \%, 95.5 \%$ and, $95.5 \%$ for histogram features, respectively (Table 3). Comparison of the non-resampled data with thick-slice images of the resampled data showed a significant decrease in texture features $(\mathrm{P}<0.001)$. When both the thick-slice images and thin-slice images were resampled, the features 
Table 2 Number of different and consistent RF values between different comparison groups

\begin{tabular}{ccccc}
\hline & Thick vs. thin (\%) & Thick-r vs. thin (\%) & Thick-r vs. thin-r (\%) & $\mathrm{P}_{1}{ }^{*}$ \\
\hline $\mathrm{P}<0.05$ & $239(78.4)$ & $202(66.2)$ & $247(81.0)$ & $<0.01$ \\
$\mathrm{P} \geq 0.05$ & $66(21.6)$ & $103(33.8)$ & $58(19.0)$ & \\
\hline
\end{tabular}

$\mathrm{P}_{1}{ }^{*}$, comparision between thick vs. thin and thick-r vs. thin; $\mathrm{P}_{2}{ }^{\star *}$, comparision between thick vs. thin and thick-r vs. thin-r. RF, radiomic feature; thick-r, thick-slice images resampled; thin-r, thin-slice images resampled.

Table 3 Number of different and consistent RF values of subgroup of RFs between different comparison groups

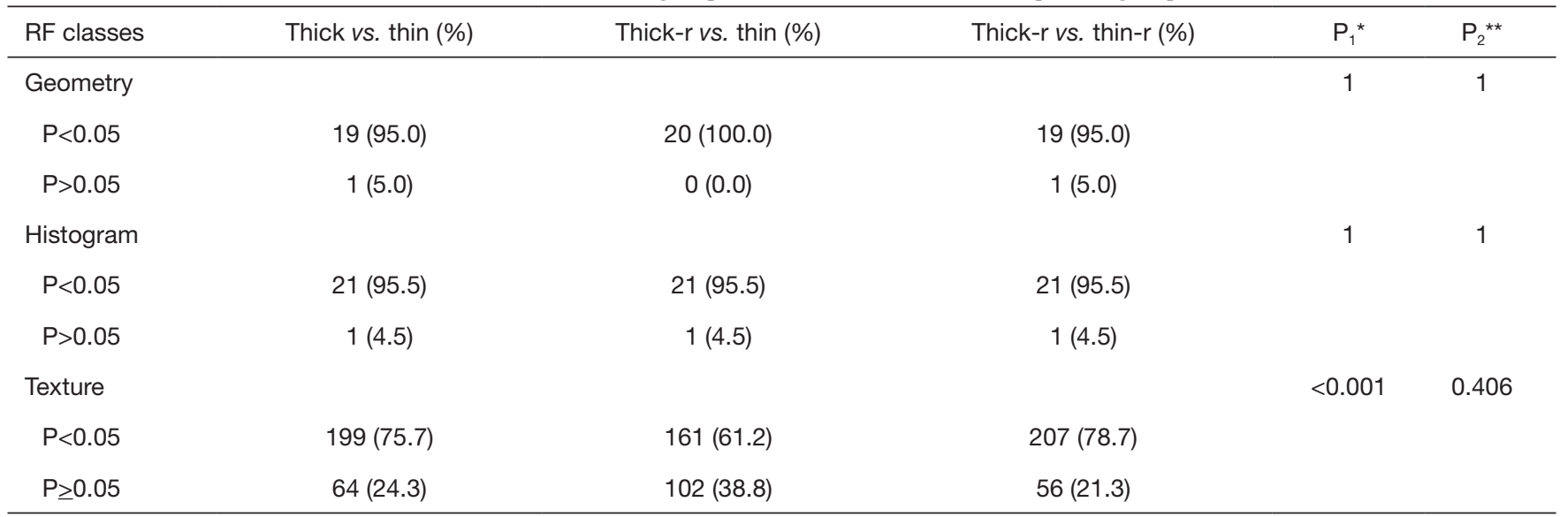

$\mathrm{P}_{1}{ }^{*}$, comparison between thick vs. thin and thick-r vs. thin; $\mathrm{P}_{2}{ }^{\star \star}$, comparision between thick vs. thin and thick-r vs. thin-r. RF, radiomic feature; thick-r, thick-slice images resampled; thin-r, thin-slice images resampled.

with significant differences increased, but without statistical significance $(\mathrm{P}=0.406)$. There were no significant changes in the geometry and histogram features (Table 3).

\section{Discussion}

In the present study, a considerable number of different RFs between thick-slice and thin-slice CT images of solid pulmonary nodules were identified, and proper resampling decreased this difference. However, resampling was insufficient to correct the influence of slice thickness, indicating that it is inappropriate to apply CT images with different thicknesses to radiomic study only using linear interpolation.

The advantage of the present study is its prospective nature; therefore, the factors that might influence the results could be controlled. Compared with phantom studies, this patient study better reflects the internal characteristics of pulmonary nodules. Besides, the subgroups of RFs were analyzed further to understand the influence of slice thickness on radiomic studies.

Our results showed that $77.0 \%$ (305 of 396) of the RFs were reproducible in the test-retest analysis. Berenguer et al. (21) observed that reproducible RFs accounted for $91.0 \%$ (161 of 177) in the test-retest analysis. Our results were similar but slightly less than those in Berenguer et al.'s study (21). This difference might be because the subjects in this study were patients, while Berenguer's study used phantoms. Previous studies $(16,21,22)$ showed that when the slice thickness changed, other parameters remained consistent, and the proportion of reproducible RFs were not high enough. These results were consistent with those of the present study, which demonstrated that even if other conditions were the same, mixing up images with different thicknesses resulted in false positives. Notwithstanding, the difference in the number of RFs caused by different slice thicknesses still should be corrected.

Resampling to a specific voxel size is one of the standard radiomic methods $(17,23)$. Shafiq-Ul-Hassan et al. (17) demonstrated that after resampling, 42 of the 213 extracted features showed significant improvement. That result was in line with our findings, indicating that resampling could reduce the difference in RFs and make them more reproducible. Besides, our results showed that the texture 
features demonstrated a relatively better reproducibility than the histogram and geometry features, and proper resampling could improve the reproducibility of texture features. The reason for this might be that the boundaries of the thick-slice and thin-slice CT images tend to differ in segmentation, resulting in poor reproducibility of the histogram and geometry features. However, these results were inconsistent with those of the study conducted by Park et al. (22). The difference between the studies might be due to diverse types of selected pulmonary nodules and different compositions of RFs. Previous studies have advocated resampling of all nodules before segmentation $(18,19)$. However, in this study, when both thick-slice and thinslice images were resampled, the number of significantly different RF values between thick-r and thin-r images increased compared with that in the non-resampled data. It is still debatable as to what kind of layer thickness image should be resampled. In addition, an oversampling method in which all images with different thicknesses are resampled is considered unsuitable for radiomic studies.

The Fleischner Society recommends a routine $5-\mathrm{mm}$ layer thickness examination and thin layer reconstruction to manage incidental pulmonary nodules (24). However, not all patients had thin-slice thickness images in the actual clinical conditions. Most of the existing radiomic studies were retrospective in nature and used a large amount of data. In view of the influence of slice thickness on RFs, pulmonary nodules with only thick slice images were usually discarded in these radiomic studies. This approach might cause a certain degree of bias, and the radiomic results obtained from thinslice images cannot necessarily be applied to thick-slice CT images. Our study showed that the resampling method could improve the reproducibility of RFs, but over resampling might increase the difference. In any case, this was not sufficient to apply different slice CT images in radiomic studies only by the linear interpolation. Further research is warranted to explore better standardization methods, to determine what kind of images require resampling, and how to select the resampled voxel size.

There are some limitations to our study. First, the number of cases in the study was relatively small, and only 30 pulmonary nodules were included because we used prospective case selection. Second, our study mainly focused on the effect of different slice thicknesses and the resampling method on RFs, and the 30 cases of pulmonary nodules demonstrated no pathological results. Third, the segmentation of the nodules in this study was performed manually. If semi-automatic segmentation was used, then the effect on the results caused by differences in segmentation might be smaller.

In conclusion, slice thickness had a large influence on the RF values in solid pulmonary nodules, producing false results when different slice thickness CT images were used. Linear interpolation of the resampling method demonstrated limited effectiveness in correcting the influence of slice thickness. Also, it is not appropriate to apply CT images of different thicknesses to study radiomics.

\section{Acknowledgments}

The author would like to thank GE Healthcare Life Sciences for its support and help in this research.

Funding: This work was supported by the National Key R\&D Program of China [grant number 2017YFC1308700]; the National Natural Science Foundation of China [grant numbers 81701692, 81771830]; and the CAMS Innovation Fund for Medical Sciences [grant number 2019-I2M-2002].

\section{Footnote}

Reporting Checklist: The authors have completed the STROBE reporting checklist. Available at http://dx.doi. org/10.21037/atm-20-2992

Data Sharing Statement: Available at http://dx.doi. org/10.21037/atm-20-2992

Conflicts of Interest: All authors have completed the ICMJE uniform disclosure form (available at http://dx.doi. org/10.21037/atm-20-2992). ML serves as an unpaid section editor of Annals of Translational Medicine. The other authors have no conflicts of interest to declare.

Ethical Statement: The authors are accountable for all aspects of the work in ensuring that questions related to the accuracy or integrity of any part of the work are appropriately investigated and resolved. The study was conducted in accordance with the Declaration of Helsinki (as revised in 2013). The study was approved by the medical ethics committee of National Cancer Center/Cancer Hospital, Chinese Academy of Medical Sciences and Peking Union Medical College (NO.: 19-018/1840) and informed consent was taken from all the patients.

Open Access Statement: This is an Open Access article 


\section{Page 8 of 9}

distributed in accordance with the Creative Commons Attribution-NonCommercial-NoDerivs 4.0 International License (CC BY-NC-ND 4.0), which permits the noncommercial replication and distribution of the article with the strict proviso that no changes or edits are made and the original work is properly cited (including links to both the formal publication through the relevant DOI and the license). See: https://creativecommons.org/licenses/by-nc$\mathrm{nd} / 4.0 /$.

\section{References}

1. Lambin P, Rios-Velazquez E, Leijenaar R, et al. Radiomics: Extracting more information from medical images using advanced feature analysis. Eur J Cancer 2012;48:441-6.

2. Rizzo S, Botta F, Raimondi S, et al. Radiomics: the facts and the challenges of image analysis. Eur Radiol Exp 2018;2:36.

3. Nougaret $\mathrm{S}$, Tibermacine $\mathrm{H}$, Tardieu $\mathrm{M}$, et al. Radiomics: an Introductory Guide to What It May Foretell. Curr Oncol Rep 2019;21:70.

4. Tan X, Ma Z, Yan L, et al. Radiomics nomogram outperforms size criteria in discriminating lymph node metastasis in resectable esophageal squamous cell carcinoma. Eur Radiol 2019;29:392-400.

5. She Y, Zhang L, Zhu H, et al. The predictive value of CT-based radiomics in differentiating indolent from invasive lung adenocarcinoma in patients with pulmonary nodules. Eur Radiol 2018;28:5121-8.

6. Hu T, Wang S, Huang L, et al. A clinical-radiomics nomogram for the preoperative prediction of lung metastasis in colorectal cancer patients with indeterminate pulmonary nodules. Eur Radiol 2019;29:439-49.

7. Liu B, Chi W, Li X, et al. Evolving the pulmonary nodules diagnosis from classical approaches to deep learning-aided decision support: three decades' development course and future prospect. J Cancer Res Clin Oncol 2020;146:153-85.

8. Mao L, Chen $\mathrm{H}$, Liang $\mathrm{M}$, et al. Quantitative radiomic model for predicting malignancy of small solid pulmonary nodules detected by low-dose CT screening. Quant Imaging Med Surg 2019;9:263-72.

9. Liu S, Liu S, Zhang C, et al. Exploratory Study of a CT Radiomics Model for the Classification of Small Cell Lung Cancer and Non-small-Cell Lung Cancer. Front Oncol 2020;10:1268.

10. Yan M, Wang W. Radiomic Analysis of CT Predicts
Yang et al. The influence of slice thicknesses on radiomic features

Tumor Response in Human Lung Cancer with Radiotherapy. J Digit Imaging 2020;33:1401-3.

11. Mackin D, Fave X, Zhang L, et al. Measuring Computed Tomography Scanner Variability of Radiomics Features. Invest Radiol 2015;50:757-65.

12. Lu L, Ehmke RC, Schwartz LH, et al. Assessing Agreement between Radiomic Features Computed for Multiple CT Imaging Settings. PLoS One 2016;11:e0166550.

13. Kim YJ, Lee HJ, Kim KG, et al. The Effect of CT Scan Parameters on the Measurement of CT Radiomic Features: A Lung Nodule Phantom Study. Comput Math Methods Med 2019;2019:8790694.

14. Zhao B, Tan Y, Tsai WY, et al. Reproducibility of radiomics for deciphering tumor phenotype with imaging. Sci Rep 2016;6:23428.

15. He L, Huang Y, Ma Z, et al. Effects of contrastenhancement, reconstruction slice thickness and convolution kernel on the diagnostic performance of radiomics signature in solitary pulmonary nodule. Sci Rep 2016;6:34921.

16. Larue RTHM, van Timmeren JE, de Jong EEC, et al. Influence of gray level discretization on radiomic feature stability for different CT scanners, tube currents and slice thicknesses: a comprehensive phantom study. Acta Oncol 2017;56:1544-53.

17. Shafiq-Ul-Hassan M, Zhang GG, Latifi K, et al. Intrinsic dependencies of CT radiomic features on voxel size and number of gray levels. Med Phys 2017;44:1050-62.

18. Shafiq-Ul-Hassan M, Latifi K, Zhang G, et al. Voxel size and gray level normalization of CT radiomic features in lung cancer. Sci Rep 2018;8:10545.

19. Mackin D, Fave X, Zhang L, et al. Harmonizing the pixel size in retrospective computed tomography radiomics studies. PLoS One 2017;12:e0178524.

20. Feng Q, Chen Y, Liao Z, et al. Corpus Callosum Radiomics-Based Classification Model in Alzheimer's Disease: A Case-Control Study. Front Neurol 2018;9:618.

21. Berenguer R, Pastor-Juan M, Canales-Vazquez J, et al. Radiomics of CT Features May Be Nonreproducible and Redundant: Influence of CT Acquisition Parameters. Radiology 2018;288:407-15.

22. Park S, Lee SM, Do KH, et al. Deep Learning Algorithm for Reducing CT Slice Thickness: Effect on Reproducibility of Radiomic Features in Lung Cancer. Korean J Radiol 2019;20:1431-40.

23. Limkin EJ, Reuze S, Carre A, et al. The complexity of tumor shape, spiculatedness, correlates with tumor 
radiomic shape features. Sci Rep 2019;9:4329.

24. MacMahon H, Naidich DP, Goo JM, et al. Guidelines for Management of Incidental Pulmonary Nodules

Cite this article as: Yang S, Wu N, Zhang L, Li M. Evaluation of the linear interpolation method in correcting the influence of slice thicknesses on radiomic feature values in solid pulmonary nodules: a prospective patient study. Ann Transl Med 2021;9(4):279. doi: 10.21037/atm-20-2992
Detected on CT Images: From the Fleischner Society 2017. Radiology 2017;284:228-43. 